04

\title{
Дрейфовый механизм формирования обдирочного слоя в токамаке
}

\section{() Е.Г. Кавеева, В.А. Рожанский}

Санкт-Петербургский политехнический университет Петра Великого, Санкт-Петербург, Россия

E-mail: Kaveeva@spbau.ru

Поступило в Редакцию 6 октября 2017 г.

Рассмотрена модель обдирочного слоя токамака (scrape-off layer, SOL) в предположении дрейфовых механизмов переноса тепла электронов и преобладания рециклинга снаружи сепаратрисы над потоком ионов из области удержания. Оценена ширина SOL. Показано, что чисто дрейфовые механизмы дают минимальную ширину SOL, подчиняющуюся современным скейлингам и близкую по порядку величины к экспериментальным значениям.

DOI: 10.21883/PJTF.2018.06.45764.17069

Ширина обдирочного слоя (scrape-off layer, SOL) в токамаке является одним из критических параметров для создания на его основе термоядерного реактора. Согласно современным скейлингам, ширина SOL $\lambda_{q}$ для электронного потока тепла в Международном экспериментальном термоядерном реакторе (ИТЭР) на внешнем обводе может быть всего $1 \mathrm{~mm}$ [1], что создает недопустимо высокую нагрузку на пластины дивертора. Экспериментальная параметрическая зависимость $\lambda_{q} \sim q_{95} / B$ от отношения запаса устойчивости на границе плазмы $q_{95}$ к магнитному полю $B$ в настоящее время может объясняться моделью Голдстона [2]. В ней ширина SOL определяется дрейфовым переносом ионов в SOL на фоне высокой электронной аномальной теплопроводности. Неявно такая модель предполагает, что источники ионизации расположены внутри сепаратрисы и магнитные трубки в SOL наполняются плазмой за счет радиального переноса выше $X$-точки. В то же время моделирование показывает, что рециклинг (объемная ионизация и последующая рекомбинация на поверхности пластин) происходит в основном в диверторной области, и потоки плазмы из SOL на его фоне для больших токамаков несущественны. В некоторых 
случаях в моделировании даже возникают потоки из диверторной области к внешнему обводу [3]. Ширина SOL определяется потоком тепла электронов, который позволяет создать плазму за счет ионизации уже внутри самих магнитных трубок в SOL. B настоящей работе изложена аналитическая модель SOL в предположении радиального переноса тепла электронов дрейфовыми механизмами. Продольный перенос тепла предполагается за счет классической теплопроводности, он рассмотрен в двух типичных режимах работы дивертора: режимах sheath-limited и conduction-limited [4]. В плазме токамака важную роль играет радиальный турбулентный перенос, однако дрейфовые механизмы обязательно присутствуют, и они определяют минимальную ширину SOL.

Режим sheath-limited - это режим с высокой температурой и вследствие этого высокой продольной теплопроводностью электронов. В нем поток тепла электронов на пластины дивертора ограничивается переносом тепла в слое пространственного заряда и магнитном предслое, а электронная температура на магнитной поверхности почти не меняется. Поэтому во всех выкладках будем считать ее функцией только радиальной координаты $y$. Рассмотрим стандартную геометрию, при которой градиентный дрейф ионов (дрейф в неоднородном магнитном поле) направлен вниз - к активной $X$-точке и пластинам дивертора. В общем виде с учетом радиальных дрейфовых слагаемых и продольной теплопроводности баланс энергии электронов в SOL записывается как [5]

$$
\nabla\left[5 / 2 n T_{e}\left(\mathbf{V}_{e}^{d i a}+\mathbf{V}_{e}^{E x B}+\mathbf{V}_{e \|}\right)+\mathbf{q}_{e}^{d i a}+\mathbf{q}_{\|}\right]=-e n \mathbf{E} \mathbf{V}_{e \|}-e n \mathbf{E} \mathbf{V}_{e}^{d i a},
$$

где $\mathbf{V}_{e}^{\text {dia }}=-\frac{1}{e n} \frac{\left[\mathbf{B} \times \nabla n T_{e}\right]}{B^{2}}, \mathbf{V}_{e}^{E x B}=\frac{1}{e n} \frac{[\mathbf{B} \times \nabla \phi]}{B^{2}}, \mathbf{q}_{e}^{d i a}=-\frac{5}{2} \frac{n T_{e}}{e} \frac{\left[\mathbf{B} \times \nabla T_{e}\right]}{B^{2}}, n-$ концентрация плазмы; $T_{e}$ - температура электронов, $\phi$ - электростатический потенциал, $q_{\|}$- продольный поток энергии. Замена диамагнитных слагаемых слагаемыми, связанными с градиентным дрейфом, дает

$$
\nabla\left[5 / 2 n T_{e}\left(\mathbf{V}_{e}^{\nabla B}+\mathbf{V}_{e \|}\right)+3 / 2 n T_{e} \mathbf{V}_{e}^{E x B}+\mathbf{q}_{\|}\right]=-e n \mathbf{E} \mathbf{V}_{e \|}-e n \mathbf{E} \mathbf{V}_{e}^{\nabla B},
$$

где $\mathbf{V}_{e}^{\nabla B}=T_{e}\left[\mathbf{B} \times \nabla B^{-2}\right] / e$.

Рассмотрим источники и дивергенции потоков, проинтегрировав их в объеме между соседними магнитными поверхностями от внутренней 
пластины 1 до внешней пластины 2 дивертора. Область интегрирования включает магнитный предслой около пластин. В этой области должны быть учтены значительные потоки, связанные с электрическим дрейфом в быстро меняющемся по направлению к пластине потенциале, возникающем из-за резкого падения концентрации электронов. Первое слагаемое в левой части дает

$$
\begin{aligned}
& \int_{1}^{2} \nabla\left[5 / 2 n T_{e} \mathbf{V}_{e}^{\nabla B}\right] \sqrt{g} d x=\int_{1}^{2} \frac{\partial}{\partial x}\left(\frac{\sqrt{g}}{h_{x}} 5 / 2 n T_{e} V_{e x}^{\nabla B}\right) d x \\
& +\int_{1}^{2} \frac{\partial}{\partial y}\left(\frac{\sqrt{g}}{h_{y}} 5 / 2 n T_{e} V_{e y}^{\nabla B}\right) d x .
\end{aligned}
$$

Здесь $x$ - полоидальная, $y$ - радиальная, $z$ - тороидальная координата, $h_{x}, h_{y}, h_{z}$ - коэффициенты Ламе, $\sqrt{g}=h_{x} h_{y} h_{z}$, система координат соответствует работе [6]. Первым слагаемым в правой части формулы (2) можно пренебречь, поскольку в магнитном предслое концентрация существенно падает [4] и на границах интегрирования оказывается на порядок меньше, чем внутри области интегрирования. Второе слагаемое - производная от потока тепла, связанного с градиентным дрейфом по радиальной координате. Оно может быть выписано в явном виде в предположении, что концентрация плазмы меняется в узком слое перед пластинами, где происходит ионизация, и появляется поток плазмы к пластине, а в основном объеме магнитной трубки концентрация остается постоянной:

$$
\begin{aligned}
& \int_{1}^{2} \nabla\left[5 / 2 n T_{e} \mathbf{V}_{e}^{\nabla B}\right] \sqrt{g} d x=\frac{\partial}{\partial y} \int_{1}^{2}\left(\frac{5}{2} n T_{e}^{2} \frac{B_{z} h_{z}}{e} \frac{\partial B^{-2}}{\partial x}\right) d x \\
& \approx \frac{\partial}{\partial y}\left[\frac{5}{2} n_{u} T_{e}^{2} \frac{B_{z} h_{z}}{e}\left(\frac{1}{B_{2}^{2}}-\frac{1}{B_{1}^{2}}\right)\right] .
\end{aligned}
$$

Здесь и далее индекс $и$ обозначает часть магнитной трубки вдали от пластин (,upstream“), индекс $t$ обозначает пластину („target“"). Слагаемое в левой части уравнения (1b), связанное с электрическим дрейфом, можно преобразовать аналогично, и затем подставить в

Письма в ЖТФ, 2018, том 44, вып. 6 
него полоидальное электрическое поле, выраженное через продольный баланс сил для электронов:

$$
\begin{gathered}
-\frac{\partial \phi}{\partial x}=-\frac{1}{e n} \frac{\partial n T_{e}}{\partial x}-\frac{0.71}{e} \frac{\partial T_{e}}{\partial x}, \\
\int_{1}^{2} \nabla\left[3 / 2 n T_{e} \mathbf{V}_{e}^{E x B}\right] \sqrt{g} d x \approx \frac{\partial}{\partial y} \int_{1}^{2}\left(\frac{\sqrt{g}}{h_{y}} 3 / 2 n T_{e} V_{e y}^{E x B}\right) d x \\
\approx \frac{\partial}{\partial y} \int_{1}^{2}\left(\frac{3}{2} T_{e}^{2} \frac{B_{z} h_{z}}{e B^{2}} \frac{\partial n}{\partial x}\right) d x .
\end{gathered}
$$

Снова пользуясь предположением, что концентрация плазмы меняется в узком слое перед пластинами дивертора, получаем

$$
\int_{1}^{2} \nabla\left[3 / 2 n T_{e} \mathbf{V}_{e}^{E x B}\right] \sqrt{g} d x \approx-\frac{\partial}{\partial y}\left[\frac{3}{2} n_{u} T_{e}^{2} \frac{B_{z} h_{z}}{e}\left(\frac{1}{B_{2}^{2}}-\frac{1}{B_{1}^{2}}\right)\right] .
$$

Оценим интеграл дрейфового слагаемого $-e n \mathbf{E V}_{e}^{\nabla B}$ в правой части уравнения (1b)

$$
\begin{aligned}
& -\int_{1}^{2} e n \mathbf{E V}_{e}^{\nabla B} \sqrt{g} d x=\int_{1}^{2} h_{z} B_{z} n T_{e}\left[-\frac{\partial B^{-2}}{\partial y} \frac{\partial \phi}{\partial x}\right] d x \\
& +\int_{1}^{2} h_{z} B_{z} n T_{e}\left[\frac{\partial B^{-2}}{\partial x} \frac{\partial \phi}{d y}\right] d x .
\end{aligned}
$$

Чтобы оценить $\partial \phi / \partial y$, нужно учесть перепад потенциала в слое и предслое и затем проинтегрировать уравнение $(4), \phi \approx \frac{T_{e}}{e}\left(3+\ln \frac{2 n}{n_{u}}\right)$. Первое слагаемое в правой части (7) можно оценить как

$$
I_{1}=\int_{1}^{2} h_{z} B_{z} n T_{e}\left[-\frac{\partial B^{-2}}{\partial y} \frac{\partial \phi}{\partial x}\right] d x \sim \frac{h_{z} B_{z} n T_{e}^{2}}{e} \frac{\partial B^{-2}}{\partial y},
$$

Письма в ЖТФ, 2018, том 44, вып. 6 
второе - как

$$
I_{2}=\int_{1}^{2} h_{z} B_{z} n T_{e}\left[\frac{\partial B^{-2}}{\partial x} \frac{\partial \phi}{\partial y}\right] d x \approx-n T_{e} \frac{B_{z} h_{z}}{e}(3+\ln 2) \frac{\partial T_{e}}{\partial y}\left(\frac{1}{B_{2}^{2}}-\frac{1}{B_{1}^{2}}\right) .
$$

Второе слагаемое больше, $I_{1} / I_{2} \sim \frac{L_{y}}{r}$, поэтому в дальнейшем не будем обсуждать $I_{1}$. Теперь соберем в правой части дрейфовые слагаемые в балансе тепла, а в левой оставим слагаемые, связанные с продольным переносом:

$$
\begin{aligned}
& \int_{1}^{2} \nabla\left[5 / 2 n T_{e} \mathbf{V}_{e \|}+\mathbf{q}_{\|}\right] \sqrt{g} d x=\int_{1}^{2} \frac{\partial}{\partial x}\left(\frac{\sqrt{g}}{h_{x}} b_{x}\left[5 / 2 n T_{e} V_{e \|}+q_{\|}\right]\right) d x \\
& =\frac{B_{z} h_{z}}{e} n_{u} T_{e}(3+\ln 2) \frac{\partial T_{e}}{\partial y}\left(\frac{1}{B_{2}^{2}}-\frac{1}{B_{1}^{2}}\right)-\frac{B_{z} h_{z}}{e} \frac{\partial n_{u} T_{e}^{2}}{\partial y}\left(\frac{1}{B_{2}^{2}}-\frac{1}{B_{1}^{2}}\right) .
\end{aligned}
$$

Для оценки радиального масштаба можно использовать

$$
\begin{aligned}
& \left.\frac{\sqrt{g}}{h_{x}} b_{x}\left[5 / 2 n T_{e} V_{e \|}+q_{\|}\right]\right|_{1} ^{2}=\frac{B_{z} h_{z}}{e} n_{u} T_{e} \\
& \times\left[(1+\ln 2) \frac{\partial T_{e}}{\partial y}-\frac{T_{e}}{n_{u}} \frac{\partial n_{u}}{\partial y}\right]\left(\frac{1}{B_{2}^{2}}-\frac{1}{B_{1}^{2}}\right) .
\end{aligned}
$$

Согласно граничному условию для переноса тепла в слое, пренебрегая термоэлектрическим током, получим $5 / 2 n T_{e} V_{e \|}+q_{\|} \approx \pm \gamma n T_{e} \sqrt{T_{e} / m_{i}}$, где $\gamma \sim 5-6$ - коэффииент трансмиссии в слое [4], и в режиме sheath-limited $n_{t}=n_{u} / 2$. Учитывая сохранение полоидального магнитного потока $B_{x} h_{y} h_{z}=$ const, получим

$$
\begin{aligned}
& \frac{\gamma}{2} n_{u} T_{e} \sqrt{T_{e} / m_{i}}\left(\frac{1}{B_{2}}+\frac{1}{B_{1}}\right)=\frac{B_{z}}{e B_{x}} n_{u} T_{e} \\
& \times\left[(1+\ln 2) \frac{\partial T_{e}}{h_{y} \partial y}-\frac{T_{e}}{n_{u}} \frac{\partial n_{u}}{h_{y} \partial y}\right]\left(\frac{1}{B_{2}^{2}}-\frac{1}{B_{1}^{2}}\right) .
\end{aligned}
$$

Выражение в правой части не зависит от изменения площади магнитной трубки вдоль ее длины. Можно предположить, что электронная

Письма в ЖТФ, 2018, том 44, вып. 6 
температура и концентрация меняются на сравнимых масштабах, поскольку распределение концентрации зависит от ионизации в SOL, а она в свою очередь велика только при большой электронной температуре. Тогда для $L_{y}$ получаем оценку

$$
L_{y}=\frac{B_{z}}{e B_{x}} \frac{2}{\gamma} \sqrt{T_{e} m_{i}}\left(\frac{1}{B_{2}^{2}}-\frac{1}{B_{1}^{2}}\right) /\left(\frac{1}{B_{2}}+\frac{1}{B_{1}}\right),
$$

в которой $B_{x}, B_{z}$ нужно брать для той же полоидальной координаты, где измеряется радиальный масштаб. Упрощая выражение (11), получаем тот же масштаб, который был определен в работе [2]

$$
L_{y} \approx \frac{4}{\gamma} q \rho_{c i} \approx q \rho_{c i}
$$

Ту же оценку можно получить, используя баланс тепла, включающий бездивергентную часть диамагнитного потока частиц и тепла [4].

Отдельно можно качественно рассмотреть довольно обычную ситуацию, когда длина свободного пробега электрона сравнима с длиной магнитной трубки. В этом случае электроны, стартовавшие с большой энергией на внешнем обводе на сепаратрисе, свободно долетают до пластин дивертора. Но эти электроны не смогут попасть на пластину, если не обеспечен равный по величине поток ионов. Электрон будет отражен электрическим полем у пластин и будет двигаться между пластинами до тех пор, пока он не произведет в среднем одну ионизацию. Все это время он будет двигаться под действием градиентного и электрического дрейфа. Радиальные потоки, связанные с дрейфами, как и условие переноса тепла в слое, остаются теми же самыми и в случае малой столкновительности. Поэтому оценка для масштаба $L_{y}$ остается справедливой.

В режиме conduction-limited можно проделать аналогичные оценки. Это режим с большей плотностью и меньшей температурой плазмы. Поток тепла на пластину дивертора ограничен теплопроводностью плазмы, и характерная температура на пластинах дивертора намного меньше, чем в верхней части SOL: $T_{e t} \ll T_{e u}$. Теплопроводность электронов резко спадает с температурой и не зависит от концентрации, поэтому область резкого спада температуры находится в диверторе, где сама температура мала. Для оценок будем предполагать, что область спада температуры шире, чем область ионизации, где происходит резкое

Письма в ЖТФ, 2018, том 44, вып. 6 
изменение давления. Все выкладки можно проделать и в обратном предположении, при этом качественно результат не поменяется. Теперь вклад электрического дрейфа в поток тепла

$$
\begin{aligned}
& \int_{1}^{2} \nabla\left[3 / 2 n T_{e} \mathbf{V}_{e}^{E x B}\right] \sqrt{g} d x \approx \frac{\partial}{\partial y} \int_{1}^{2}\left(\frac{3}{2} n T_{e} \frac{B_{z} h_{z}}{B^{2}} \frac{\partial \phi}{\partial x}\right) d x \\
& \approx \frac{\partial}{\partial y} \int_{1}^{2} \frac{3}{2} \frac{B_{z} h_{z}}{e B^{2}} T_{e}\left(\frac{\partial n T_{e}}{\partial x}+0.71 n \frac{\partial T_{e}}{\partial x}\right) d x .
\end{aligned}
$$

Слагаемым в правой части, пропорциональным градиенту давления, можно пренебречь, поскольку давление меняется в области с низкой температурой электронов $T_{e} \approx T_{e t}$ :

$$
\int_{1}^{2} \nabla\left[3 / 2 n T_{e} \mathbf{V}_{e}^{E x B}\right] \sqrt{g} d x \approx-\frac{\partial}{\partial y}\left[\frac{3}{2} 0.71 n_{u} T_{e u}^{2} \frac{B_{z} h_{z}}{e}\left(\frac{1}{B_{2}^{2}}-\frac{1}{B_{1}^{2}}\right)\right]
$$

Вклад градиентного дрейфа не отличается от определенного в уравнении (3) для режима sheath-limited, поскольку области, где меняются температура и давление намного у́же, чем область изменения магнитного поля.

Интегрируя электрический потенциал по $x$, можно ввиду низкой температуры на пластинах пренебречь его перепадом в слое. Изменение потенциала из-за изменения давления тоже незначительно. Оно происходит в области с низкой температурой и вследствие этого высокой плотностью, из-за которой первое слагаемое в правой части уравнения (4) мало. Поэтому практически везде можно использовать $\phi \approx 0.71 \frac{T_{e}}{e}$.

Дрейфовое слагаемое в правой части уравнения (1b) можно тогда проинтегрировать

$$
\begin{aligned}
& -\int_{1}^{2} e n \mathbf{E V}_{e}^{\nabla B} \sqrt{g} d x \approx \int_{1}^{2} h_{z} B_{z} n T_{e}\left[\frac{\partial B^{-2}}{\partial x} \frac{\partial \phi}{\partial y}\right] d x \\
& \approx 0.71 n_{u} T_{e u} \frac{\partial T_{e u}}{\partial y} \frac{B_{z} h_{z}}{e}\left(\frac{1}{B_{2}^{2}}-\frac{1}{B_{1}^{2}}\right) .
\end{aligned}
$$

Письма в ЖТФ, 2018, том 44, вып. 6 
Окончательно собирая все дрейфовые слагаемые в правой части баланса тепла, получаем

$$
\begin{aligned}
& \gamma n_{t} T_{e t} \sqrt{T_{e t} / m_{i}}\left(\frac{1}{B_{2}}+\frac{1}{B_{1}}\right) \\
& \approx \frac{1}{e b_{x}}\left[-1.5 \frac{\partial n_{u} T_{e u}^{2}}{h_{y} \partial y}+0.71 n_{u} T_{e u} \frac{\partial T_{e u}}{h_{y} \partial y}\right]\left(\frac{1}{B_{2}^{2}}-\frac{1}{B^{2}}\right) .
\end{aligned}
$$

Можно провести оценку для характерного радиального масштаба

$$
L_{y} \approx \frac{8}{\gamma} q \rho_{c i(u)} \sqrt{\frac{T_{u}}{T_{i}}}
$$

Эта оценка дает ширину SOL бо́льшую, чем оценка (12), однако с учетом типичных отношений температур в верхней части SOL и на пластинах дивертора отличается от нее не более чем в 4-5 раз. Видно, что учет переноса энергии электронов дрейфовыми механизмами даже без дрейфового переноса ионов (здесь мы считали, что ионизация на магнитной поверхности преобладает над переносом ионов между магнитными поверхностями) дает ту же оценку для характерного размера SOL, что и эвристический скейлинг Голдстона, основанный на представлении о дрейфовом переносе ионов и аномальной электронной теплопроводности. Это особенно важно для ИТЭР, в котором, согласно результатам моделирования [3], рециклинг ионов внутри SOL будет существенно больше, чем поток ионов через сепаратрису.

Работа выполнена в Санкт-Петербургском политехническом университете Петра Великого при поддержке Российского научного фонда (грант № 17-12-01020).

\section{Список литературы}

[1] Eich T., Leonard A.W., Pitts R.A., Fundamenski W., Goldston R.J., Gray T.K., Herrmann A., Kirk A., Kallenbach A., Kardaun O., Kukushkin A.S., LaBombard B., Maingi R., Makowski M.A., Scarabosio A., Sieglin B., Terry J, Thornton A. ASDEX Upgrade Team and JET EFDA Contributors // Nucl. Fusion. 2013 V. 53. N 9. P. 093031.

[2] Goldston R.J. // Nucl. Fusion. 2012. V. 52. N 1. P. 013009.

Письма в ЖТФ, 2018, том 44, вып. 6 
[3] Krasheninnikov S.I., Kukushkin A.S., Pshenov A.A. // Phys. Plasmas. 2016. V. 23. N 5. P. 055602.

[4] Stangeby P. The plasma boundary of magnetic fusion devices. Bristol: IOP Publ., 2000. $715 \mathrm{p}$.

[5] Брагинский С.И. // Вопросы теории плазмы / Под ред. М.А. Леонтовича. М.: Госатомиздат, 1963. В. 1. С. 205-272.

[6] Rozhansky V.A., Voskoboynikov S.P., Kaveeva E.G., Coster D., Schneider R. // Nucl. Fusion. 2001. V. 41. N 4. P. 387-402.

3 Письма в ЖТФ, 2018, том 44, вып. 6 